\title{
Correction to: Short lateral posterior condyle is associated with trochlea dysplasia and patellar dislocation
}

\author{
Julien Roger ${ }^{1,2}\left(\right.$ Débastien Lustig ${ }^{2} \cdot$ Simone Cerciello $^{3} \cdot$ Carmine Fabio Bruno $^{4} \cdot$ Philippe Neyret $^{5} \cdot$ Elvire Servien $^{2,6}$
}

Published online: 13 March 2019

๑) European Society of Sports Traumatology, Knee Surgery, Arthroscopy (ESSKA) 2019

\section{Correction to: \\ Knee Surg Sports Traumatol Arthrosc (2019) 27:731-739 \\ https://doi.org/10.1007/s00167-018-5023-2}

Unfortunately, the author name was incorrectly published in the original publication as Sébatien Lustig insted of Sébastien Lustig. The author name is corrected here by this Erratum. The original article has been corrected.

Publisher's Note Springer Nature remains neutral with regard to jurisdictional claims in published maps and institutional affiliations.

The original article can be found online at https://doi.org/10.1007/ s00167-018-5023-2.

Julien Roger

julien.roger@chu-lyon.fr

Sébastien Lustig

sebastien.lustig@gmail.com

Simone Cerciello

simone.cerciello@me.com

Carmine Fabio Bruno

dott.fabiobruno@gmail.com

Philippe Neyret

philippe.neyret01@gmail.com

Elvire Servien

elvire.servien@chu-lyon.fr
1 Groupement Hospitalier Nord, Hospices civils de Lyon, Université Claude-Bernard Lyon 1, 103 Grande Rue de la Croix-Rousse, 69004 Lyon, France

2 Groupement Hospitalier Nord, Hospices civils de Lyon, Université Lyon, 103 bvd de la Croix Rousse, 69004 Lyon, France

3 Orthopaedic Surgery, Sports Medicine, Physiotherapy, Casa di Cura Villa Betania Giomi, Via Pio IV, 42, 00165 Rome, Italy

4 Department of Orthopaedic and Trauma Surgery, "Magna Graecia” University, Catanzaro, Italy

5 Center A. Trillat, Lyon, France

6 FIFA medical center of excellence, Lyon, France 